Int. J. Dev. Biol. 50: 601-610 (2006)

doi: $10.1387 / \mathrm{ijdb} .062153 \mathrm{~kb}$

Original Article

\title{
Genetic interaction between Lef1 and Alx4 is required for early embryonic development
}

\author{
KATA BORAS-GRANIC, RUDOLF GROSSCHEDL ${ }^{1}$ and PAUL A. HAMEL* \\ Department of Laboratory Medicine and Pathobiology, University of Toronto, Toronto, Canada and \\ ${ }^{1}$ Max-Planck-Institute of Immunobiology, Freiburg, Germany
}

\begin{abstract}
Lymphoid Enhancer Factor-1 (Lef1) facilitates the assembly of transcriptional regulatory complexes and mediates nuclear responses to Wnt signals. We determined previously that the mesenchymally restricted, paired-like homeodomain protein Aristaless-like 4 (Alx4) interacts with Lef1 and together alters promoter activity of candidate genes. In order to define their overlapping functions, mice deficient for both Lef1 and Alx4 activity (Lef1-//Alx $4^{/ s t D / / s t D}$ ) were produced. Whereas embryos lacking either Lef1 or Alx4 activity remain viable up to or after birth, early embryonic lethality results when both factors were absent. No viable Lef1 $/-/ A / x 4^{I s t D / I s t D}$ embryos were recovered beyond 9.5 dpc. Between E8.5 and E10, viable Lef1 $/ / / A / x 4^{I s t D} / /$ stD embryos were developmentally delayed 0.5 days relative to littermates of all other genotypes. Principle among the alterations seen in $L e f 1^{\%} / A / x 4^{I s t D / I s t D}$ animals was defective vasculature in both embryonic and extra-embryonic tissues. In the yolk sac, while the vascular network is present, it were greatly diminished and large vitelline vessels were largely absent. Platelet/endothelial cell adhesion molecule (PECAM) staining revealed that the major vessels in the head of compound mutant embryos were absent, while the other vessels were finer than those seen in normal littermates. Pools of blood and pericardial effusion were also apparent in Lef1 $/ / / A / x 4^{/ s t D / / s t D}$ animals, further indicative of a defective vasculature. These data confirm genetically the interaction between Lef1 and Alx4 and further reveal unknown, overlapping roles for these transcription factors in embryonic vasculogenesis.
\end{abstract}

KEY WORDS: Lef1, Alx4, vasculature, yolk sac, embryonic lethal

\section{Introduction}

Organogenesis depends on the precise temporal and spatial coordination of signaling networks. The sequential and reciprocal exchange of signals occurring between different cell fields results in alteration of gene expression leading to the generation of distinct, terminally differentiated cells. Specific transcription factors provide the link between signal input and signal generation in reciprocal signaling cascades by mediating the response to inductive signals and regulating the expression of downstream signaling factors.

Lymphoid Enhancer Factor (Lef1) (Travis et al., 1991), a member of the Lef1/Tcf subfamily of high mobility group (HMG) domain-containing proteins (Giese et al., 1991), regulates gene expression in response to Wnt signals and integrates a number of signaling cascades required for the development of embryonic and postnatal tissues (de Lau and Clevers, 2001). During murine embryogenesis, Lef1 is required for the formation of tissues that depend on inductive interactions between epithelial and mesen- chymal tissues (van Genderen et al., 1994). Targeted inactivation of Lef1, which results in perinatal death, blocks the development of multiple ectodermal appendages, such as teeth, hair follicles, whisker follicles and mammary glands, at rudimentary stages. Impaired hippocampus development and generation of dentate gyrus granule cells are also evident in these Lef1-deficient animals (Galceran et al., 2000).

Lef1 is a sequence-specific DNA-binding protein whose activity is modulated via cooperative interactions with non-DNAbinding cofactors (Carlsson et al., 1993; Giese and Grosschedl, 1993; Giese et al., 1995). In response to the binding of Wnt proteins to Frizzled (Fzd) receptors, the $\beta$-catenin protein becomes stabilized, translocates to the nucleus and binds directly to the N-terminus of Lef1 (for review, see the Wnt Homepage; http:/ /stanford.edu/ rnusse/wntwindow.html). This complex, in association with additional proteins, Bcl9 and Pygopus, activates

Abbreviations used in this paper: Alx, aristaless-like; Fzd, frizzled; lef, lymphoid enhancer factor; PECAM, platelet/endothelial cell adhesion molecule. 
transcription of Wnt target genes (Behrens et al., 1996; Huber et al., 1996; Brunner et al., 1997; Kramps et al., 2002). In the absence of Wnt signaling, Groucho (Gro) binds Lef1, conferring transcriptional repression activity upon this Lef1-mediated complex (Cavallo et al., 1998; Levanon et al., 1998; Roose et al., 1998). Lef1 also functions as an architectural component in the assembly of other multiprotein enhancer complexes, such as ALY (Bruhn et al., 1997), Smad4 (Labbe et al., 2000), c-myb and Pax5 (Jin et al., 2002). Thus, while most often described in the context of the Wnt-signaling, Lef1 facilitates the integration of multiple signal transduction pathways by virtue of its ability to complex factors other than $\beta$-catenin.

We demonstrated previously that the paired-like homeodomaincontaining protein, Aristaless-like 4 (Alx4), could interact with Lef1 and synergize in the regulation of the N-CAM promoter (Boras and Hamel, 2002). Consistent with this observation is the overlapping expression of Lef1 and Alx4 in embryos (Hudson et al., 1998) in the mesenchymal compartment of a number of tissues whose development depends on Lef1 (van Genderen et al., 1994). At $9.5 \mathrm{dpc}, A / x 4$ is expressed in dorsal mesoderm adjacent to the neural tube, in the mesoderm of the first branchial arch and in mesenchymal cells of the facial region ( $\mathrm{Qu}$ et al., 1997a; Qu et al., 1997b). Later, Alx4 expression is observed specifically in mesenchymal condensations of developing hair follicles, whisker follicles and teeth as well as in cells surrounding developing cranio-facial skeletal structures (Qu et al., 1997a; Qu et al., 1997b; Hudson et al., 1998; Qu et al., 1999). Mice homozygous for the DNA-binding-defective allele of Alx4, Strong's luxoid (/st') (Forsthoefel et al., 1966; Forsthoefel, 1968; Qu et al., 1998), exhibit temporary dorsal alopecia, polydactyly on all limbs, altered

E8.5

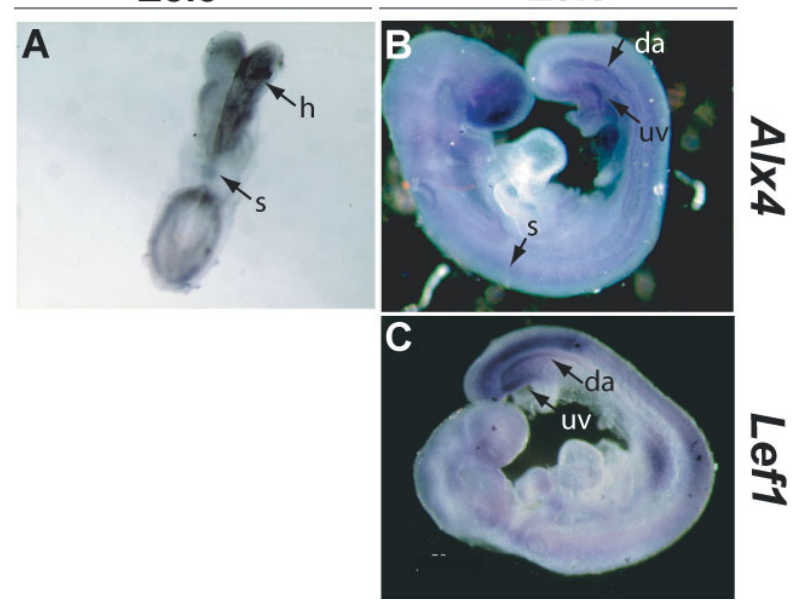

Fig. 1. Expression pattern of Alx4 and Lef1 during development. Whole-mount in situ hybridization experiments were performed to analyze the expression pattern of Alx4 and Lef1 during $8.5 \mathrm{dpc}$ and $9.5 \mathrm{dpc}$ embryonic development. (A) During $8.5 \mathrm{dpc}$, Alx4 expression is within the cephalic mesenchyme of the head, in somites and the body wall of the tail region. (B) By 9.5 dpc, expression is strongest in the craniofacial region and weaker expression is detected in the mesenchyme of the body wall. Alx4 can also be seen in the tail within the dorsal aorta and umbilical vessels. By $9.5 \mathrm{dpc}$, strong Lef1 expression is detected in the presomitic mesoderm, dorsal aorta and umbilical vessels in the tail region, as well as in the forelimb bud (C). Weaker expression is detected in the head. $h$, head fold; s, somites; uv, umbilical veins; da, dorsal aorta. cranio-facial development and, in $95 \%$ of cases, a failure in body wall closure (Qu et al., 1997b; Qu et al., 1998). We demonstrated that a proline-rich region in the $\mathrm{N}$-terminal domain of Alx4 is responsible for mediating complex formation with Lef1 through its $\mathrm{HMG}_{\text {box }}$ DNA-binding domain (Boras and Hamel, 2002). Lef1 and Alx4 bind simultaneously to a DNA sequence from the N-CAM promoter containing adjacent DNA-binding consensus sequences (Giese et al., 1991; Travis et al., 1991; Giese et al., 1992; Wilson et al., 1993; Wilson and Desplan, 1995) for Lef1 and paired-like homeodomains. These two factors also coordinately regulate $\mathrm{N}$ CAM promoter activity through these two sites.

In order to characterize the role of the interaction between Lef1 and Alx4, a genetic approach was employed in which mice lacking both Alx4 and Lef1 activity were generated. We demonstrate here that, unlike the knockout and mutant animals individually lacking Lef1 or Alx4 function and which survive to term, simultaneous loss of Alx4 and Lef1 activity results in embryonic lethality prior to 10 dpc. This lethality is associated with defective vasculogenesis. These data demonstrate a genetic interaction between Alx4 and Lef1 revealing previously unknown, overlapping functions during early mouse development.

\section{Results}

\section{Lef1 and Alx4 deficiency results in embryonic death at mid- gestation}

We demonstrated previously the overlapping expression during embryonic development of Lef1 and $A / x 4$ (Hudson et al., 1998) and their ability to form co-complexes which regulate promoter activity of target genes (Boras and Hamel, 2002). In order to further characterize the overlapping functions of Lef1 and $\mathrm{Alx} 4$, the genetic interaction between them was studied using mice deficient for both $A / \times 4$ and Lef1.

C57Bl/6 mice heterozygous for a null Lef1 allele $\left(L e f 1^{+/}\right)$ (van Genderen et al., 1994) and simultaneously heterozygous for the DNA-binding-deficient $/ s t^{D}$ allele of $A / x 4\left(A / \times 4^{+/ / s t D}\right)(\mathrm{Qu}$ et al., 1998) were crossed generating compound homozygous animals. Since compound homozygous $L$ ef $1^{-/} / A / x 4^{/ s t D / / s t D}$ animals were never observed at term (KBG, unpublished), embryos were analyzed from heterozygous intercrossings at various gestational stages. Viable $L$ ef $\gamma^{-/} / A / \times 4^{/ / S t D / / s t D}$ mutants were obtained at the expected Mendelian frequencies until $9.5 \mathrm{dpc}$ (Table 1). No viable $L e f \gamma^{-/} / A / \times 4^{/ s t D / / s t D}$ embryos were observed beyond $10 \mathrm{dpc}$. Occasional compound homozygous mutant embryos were recovered at $11.5 \mathrm{dpc}$ and $12.5 \mathrm{dpc}$. However, these embryos were invariably partially resorbed and clearly not viable. A number of empty deciduas were also recovered between $11 \mathrm{dpc}$ and $15.5 \mathrm{dpc}$, due likely to resorption of mutant embryos at earlier stages of gestation. Embryos with all other genotypes (Lef1/ / $/ A / \times 4^{+/ / s t D}$, Lef1 ${ }^{+/} / A / \times 4^{/ s t D / / s t D}, A / \times 4^{/ s t D / / s t D}$ and Lef $1^{-}$-) were viable up to at least $18.5 \mathrm{dpc}$. Thus, simultaneous loss of Alx4 and Lef1 activity results in embryonic lethality prior to $10 \mathrm{dpc}$, thus revealing a genetic interaction between these transcription factors in the post-implantation embryo.

\section{Overlapping expression of Lef1 and Alx4 in the early em- bryo}

The early lethality observed for Lef1 $/ / A / x 4^{/ s t D / / s t D}$ embryos was not predicted based on the previously determined expres- 


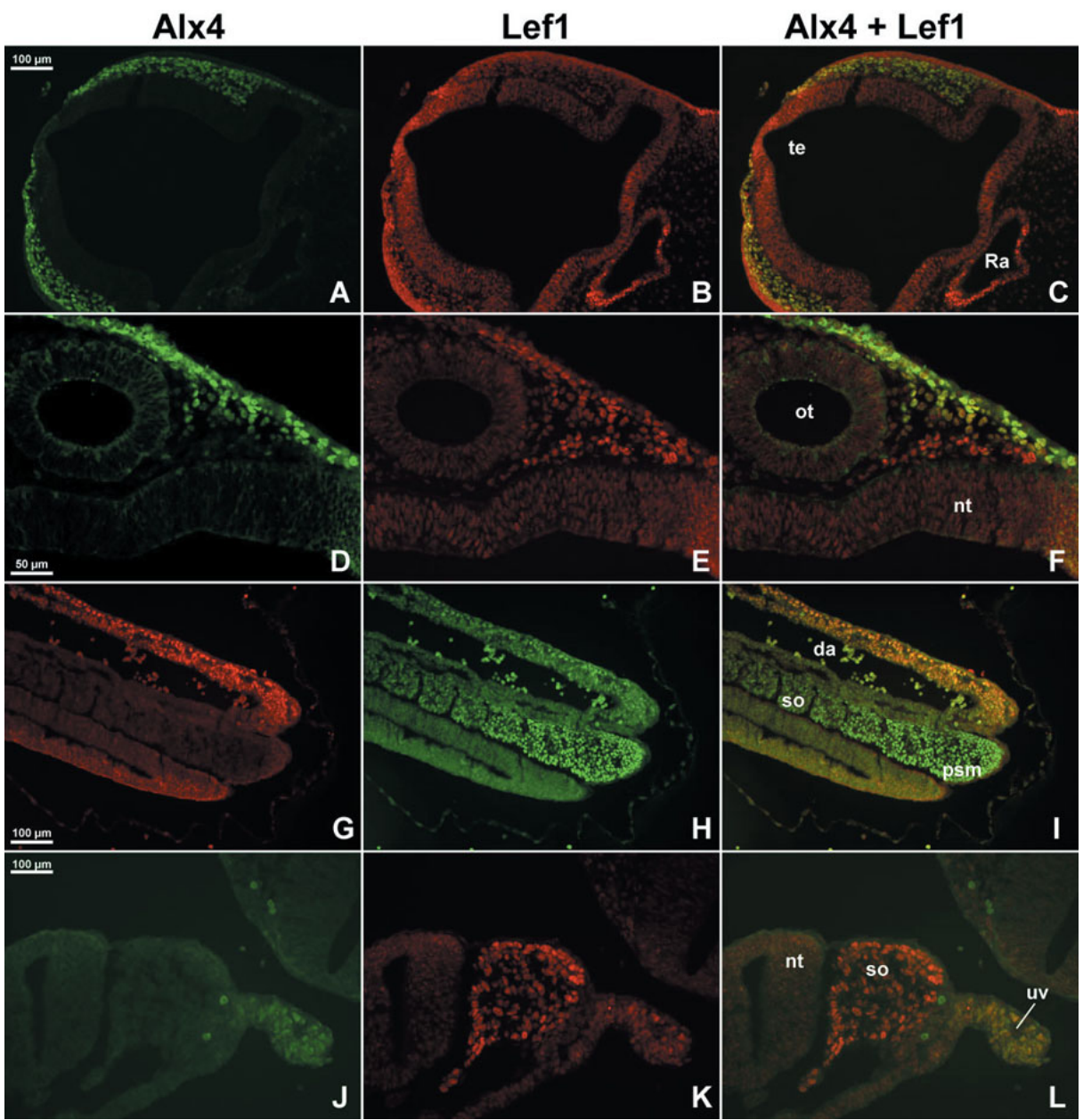

Fig. 2. Immunofluorescent detection of expression of Lef1 and Alx4. Overlapping expression of Alx4 and Lef1 was determined by immuofluorescence on $9 \mathrm{dpc}$ frozen sections. (A-C) In transverse sections, strong expression for Alx4 in the mesenchyme surrounding the telencephalic (te) vesicle is seen to overlap with Lef1 in this region. Lef1 is also expressed in Rathke's Pouch (Ra) and the cells of the ectoderm. (D-F) Sagittal sections reveal Alx4 is expressed in a tract of cells dorsal to the otic vesicle (ot) while Lef1 is expressed in this tract as well as cells medial to the otic vesicle. Staining for Lef1 in nuclei of cells in the neural tube (nt) is also evident. (G-I) In Sagittal sections of the tail region, Lef1 is strongly expressed in the presomitic mesoderm (psm) and in somites (so). Weaker but significant expression is seen on the mesenchyme ventral to the somites as well as the ventral surface of the embryo adjacent to the dorsal aorta (da). In contrast, Alx4 expression overlaps Lef1 in mesenchymal cells adjacent to the dorsal aorta. At this level, Alx4 expression is not observed in somites. (J-L) Transverse sections illustrate that $A / x 4$ and Lef1 expression overlap in the mesenchymal cells surrounding the umbilical vein. In mesenchymal cells outside of this region, Alx4 is not expressed while Lef1 expression is reduced. Considerably stronger Lef1 expression is observed in the somites adjacent to the neural tube. Bars in (A-F) and (J-L), 100 $\mu \mathrm{m}$, Bars in (D-E), $50 \mu \mathrm{m}$.

sion patterns of $A / x 4$ and Lef1 and the known defects which have been described for the individual homozygous ( $L e f 1^{-1}$ and $A / \times 4^{(s t D / s t D}$ ) embryos (van Genderen et al., 1994; Qu et al., 1997b; Qu etal., 1998; Galceran et al., 1999). Specifically, Lef1 null animals survived only until birth while the majority of $A / \times 4^{\text {stD } / s t D}$ embryos were recovered until $18.5 \mathrm{dpc}$. A small percentage $(2 \%)$ survive beyond birth and are viable and fertile. Although the known overlapping expression patterns of Lef1 and $A / x 4$ in several developing tissues during organogenesis have been examined (Hudson et al., 1998), given the embryonic lethality of the phenotype in mid-gestation, we analyzed the expression of mRNA for both $A / \times 4$ and Lef1 in embryonic tissues in situ hybridization (Fig. 1). Alx4 message was detected in mouse embryos at $8.5 \mathrm{dpc}$ (Fig. $1 \mathrm{~A})$, with expression observed, as previously shown, in the cephalic mesenchyme of the prospective craniofacial region the mesenchyme of the body wall. However, we also detected $A / \times 4$ expression in mesenchymal cells in the tail region, along the dorsal aorta as well as in somites. By 9.5 $\mathrm{dpc}$ and as described previously (Qu et al., 1997a; Qu et al., 1997b; Hudson et al., 1998), $A / x 4$ expression is detected in craniofacial mesenchyme, in the first branchial arch and in mesenchymal cells in the fore- limb bud field (Fig. 1B). Additionally, we observed $A / x 4$ expression along the dorsal aorta and umbilical vessels in the tail as well as in a restricted set of somites (typically somites 5 to 10 in animals with approximately $18-20$ somites). As described previously (Galceran et al., 1999), Lef1 expression at $9.5 \mathrm{dpc}$ is detected in the primitive streak and unsegmented presomitic mesoderm, the forelimb bud and the first branchial arch (Fig. 1C). Low levels of expression are also detected in areas of the craniofacial region. Similar to $A / x 4$, Lef1 expression is also observed in a restricted domain along the dorsal aorta and umbilical vessels in the tail.

Detailed analysis of the overlapping expression of Alx4 and Lef 1 in the early embryo $(9.0 \mathrm{dpc})$ was determined by co-

TABLE 1

\section{GENOTYPES OF OFFSPRING OF COMPOUND HETEROZYGOUS MATINGS} $L E F 1^{+/-} A L X 4^{+/ L S T D} \times L E F 1^{+/-} A L X 4^{+/ L S T D}$

\begin{tabular}{|c|c|c|c|c|c|}
\hline Age & Wild-type & $\begin{array}{l}\text { Lef1 }^{+/-}, \text {Alx4 }^{+/ / \text {stD }} \\
\text { Lef1 }^{+/ / /} / \mathbf{A l x}_{4}{ }^{+/ \text {stD }}\end{array}$ & $\begin{array}{l}\text { Lef1 }^{-/-} \\
\text {Lef1 }^{-/ / /} / \mathrm{Alx}^{+/ / \text {stD }}\end{array}$ & $\begin{array}{l}\text { Alx4 } 4^{\text {stD/lstD }} \\
\text { Lef1 }^{+/ / /} \text {Alx4 } 4^{\text {stD } / \text { stD }}\end{array}$ & Lef1 $1^{-/ /} / A \mid x 4^{1 s t D / l s t b}$ \\
\hline $9.0-9.5$ & $13 / 99(6)$ & $42 / 99(50)$ & $15 / 99$ (19) & 21/99 (19) & $8 / 99(6)$ \\
\hline$>10$ & $4 / 66(4)$ & $36 / 66(33)$ & $13 / 66(12)$ & $13 / 66(12)$ & $0 / 66$ \\
\hline
\end{tabular}

Phenotypes of $L e f 1^{+/}, A / x 4^{+/ / s t D}$, $L e f 1^{+/} / A / x 4^{+/ / s t D}$ were as wildtype; $L e f 1^{\%}$ and $L e f 1^{1 /} / A / x 4^{+/ / s t D}$ were similar;

$A / \times 4^{/ s t D / / s t D}$ and $L e f 1^{+1} / A / \times 4^{\mid s t D / / s t D}$ were similar.

Ratios represent actual numbers of embryos. Numbers in parentheses represent expected number of embryos. 
immunofluorescence (Fig. 2). Generally, Lef1 expression is observed in a wide pattern throughout both epithelial and mesenchymal structures. In contrast, Alx4 is restricted to specific mesenchymal cells. So, for example, expression of Alx4 in
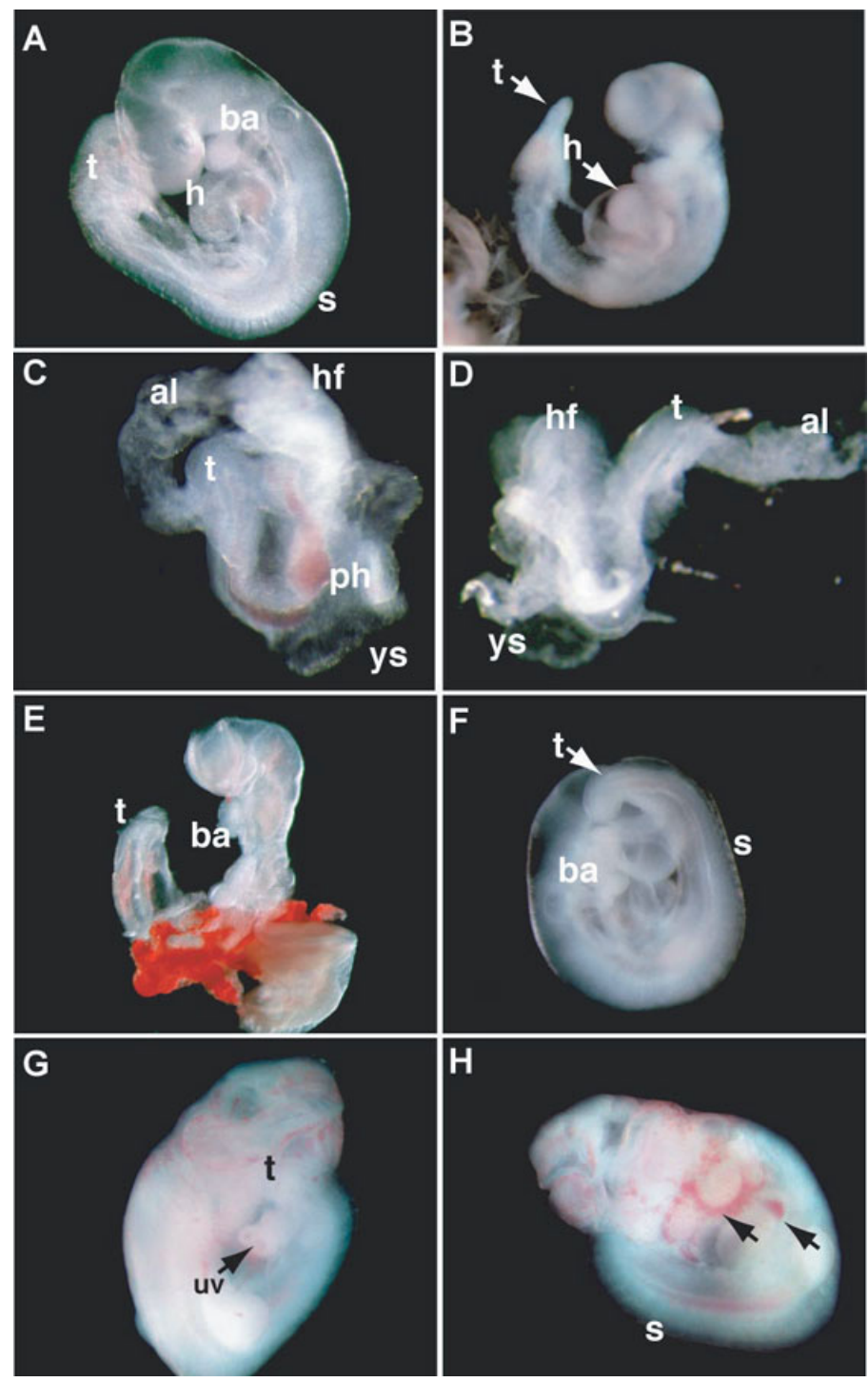

Fig. 3. Phenotypic heterogeneity of Lef1///Alx $4^{I s t D / I s t D}$ embryos. Development and morphology of control and Lef1 ${ }^{-/} / \mathrm{Al} / \mathrm{X} 4^{\mathrm{IstD} / \mathrm{stD}}$ embryos. Photographs showing $9.5 \mathrm{dpc}$ control (A) and Lef1-//Alx4 $4^{\mathrm{stD} / \mathrm{stD}}$ embryos (C-H). By 10 dpc, double homozygous embryos were no longer viable (B). The heart was smaller relative to wild type embryos and showed extensive liquid accumulation in the pericardial cavity (arrow). Also note the abnormal tail. All mutants were smaller than control littermates. Lef11-/Alx4|stD/stD embryos at $9.5 \mathrm{dpc}$ recovered were developmentally delayed [compare (A) $(28$ somites) with (C) (no somites) and (D)(3-4 somites)], more closely resembling 8-8.5 dpc embryos. (E) A mutant embryo which is slightly delayed developmentally but exhibits an open abdominal body wall with extruded abdominal organs. Another mutant embryo (G-H) displays accumulation of blood in several areas indicating potential vascular defects. Also note the enlarged umbilical region in the same embryo (G). However, more normal mutants were also recovered (F), but were invariably smaller than controls and had abnormal tails. All mutants exhibited impaired craniofacial structures and brain development. the mesenchyme surrounding the telencephalic vesicle (te) overlaps with Lef1 (Fig. 2A-C). Alx4 expression is also restricted to a potential neural crest cell migratory tract which follows a path dorsal to the otic vesicle (ot; Fig. 2D-F). Overlapping expression of Lef1 is observed in this tract. However, Lef1 expression is also present in cells between the otic vesicle and the neural tube (nt) as well as in nuclei of the neural tube and otic vesicle. In the tail region, Alx4 is highly restricted to mesenchymal cells on the ventral portion of the dorsal aorta (da; Fig. 2G) and the umbilical vein (uv; Fig. 2J). In both regions, Lef1 expression overlaps that of $\mathrm{Alx} 4$ (Fig. $2 \mathrm{H}-\mathrm{I}$ and $\mathrm{K}-\mathrm{L}$, respectively). However, it is again evident that expression of Lef1 is considerably wider than that of Alx4. The fluorescence signal for Lef1 also appears to vary considerably in specific structures at this stage. In particular, somites (so) and the presomitic mesoderm (psm) have the strongest signals, in agreement with the message levels detected by in situ hybridization (see Fig. 1C). Weaker expression for Lef1 is observed for other epithelial structures, such as the neural tube and in mesenchymal cells. Thus, overlapping expression of Alx4 and Lef1 is observed at $9.0 \mathrm{dpc}$ in a number of regions in the early embryo, this overlap restricted specifically to mesenchymal cells.

\section{Phenotype of Lef1/-/Alx $4^{\text {IstD/IstD }}$ embryos}

As exemplified in Fig. 3B, Lef1\%/A/x $4^{/ \text {stD } / / s t D}$ embryos were invariably dead at mid-gestation. Mutants were smaller than their viable littermates (Fig. 3A) and appeared developmentally delayed, although somites were evident. The tail region of these embryos was also misshaped, narrowing to a point and often exhibiting a "hook", similar to phenotypes of other mutant embryos lacking both $L e f 1$ and $T c f 1$, the latter a closely related protein of the Lef1/Tcf family that is co-expressed with Lef1 in the presomitic mesoderm (Galceran et al., 1999; Galceran et al., 2001). Fifty percent of non-viable $L$ ef $1^{-1} / A / X 4^{/ s t D / / s t D}$ embryos presented with hemorrhages in the heart, head and the dorsal vessels. Extensive accumulation of fluid in the pericardial cavity, consistent with increased permeability of cardiac microvasculature, was also typically observed (Fig. 4B and data not shown).

At earlier stages $(<10 \mathrm{dpc})$, viable compound mutant embryos, determined by a beating heart, were invariably developmentally delayed by 0.5 days relative to their littermates while exhibiting a variation in defects (Fig. 3C-I). For example, relative to normal $9.0 \mathrm{dpc}$ littermates, some $L$ ef $1^{-/ /} / A / \times 4^{/ s t D / / s t D} \mathrm{em}$ bryos resembled an $8 \mathrm{dpc}$ embryo where the head folds had not closed, the embryo had not "turned" nor had any somites developed (Fig. 3C). In contrast to the compound Lef1/Tcf1 mice (Galceran et al., 1999), somite development does not appear to be impaired in the absence of both Lef1 and $A / \times 4$ since, as seen for the $9 \mathrm{dpc}$ embryo in Fig. 3D, 4 somites were

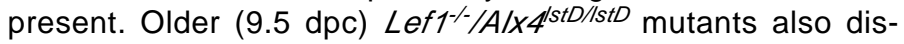
played ventrally extruded internal abdominal organs (Fig. 3E) and umbilical hernias were detected in a proportion of others (Fig. 3G). Viable embryos at all stages routinely exhibited abnormal pools of blood throughout the animal despite a clearly beating heart (Figures $3 \mathrm{E}, \mathrm{G}$ and $\mathrm{H}$ ). For example, pools of blood are evident in the cranial region and randomly throughout the body. As depicted in Fig. 3F, we recovered a single com- 
pound Lef1- $/ A / \times 4^{/ s t D / / s t D}$ embryo which appeared phenotypically relatively normal at $9.5 \mathrm{dpc}$. This mutant was slightly smaller than its littermates and exhibited a "hooked" tail.

\section{Extra-embryonic defects in Lef1 ${ }^{-/ / A} / \mathrm{x} 4^{\text {IstD/lstD }}$ embryos}

Lethality at mid-gestation often results from abnormalities in extra-embryonic tissues (Barak et al., 1999; Adams et al., 2000). Associated with non-viable $L$ ef $1^{-1} / A / \times 4^{\text {stD } / \text { st }}$ embryos at $10.5 \mathrm{dpc}$ was a severe reduction or absence of an observable vascular network and large vitelline vessels in the visceral yolk sac. Pools of free fetal blood in the amniotic cavity were observed as was evidence of intraembryonic hemorrhage in some embryos (data not shown).

Viable $L e f^{-1 /} / A / \times 4^{I^{s t D} / / s t D}$ embryos $(<10 \mathrm{dpc})$ exhibited a striking reduction in yolk sac vasculature. Specifically, yolk sacs from wild type embryos have an extensive and highly organized vasculature filled with blood by $9.5 \mathrm{dpc}$. Large vitelline vessels and numerous smaller vessels are clearly evident in the yolk sacs at this stage (Fig. 4A). In contrast, the major vessels in yolk sacs of compound homozygous $L e 1^{-1-} A / \times 4^{/ s t D / I s t D}$ embryos (Fig. 4B and C) appeared greatly reduce in size although network of branches could still be discerned (Fig. 4D).

In order to ascertain whether Lef1 and Alx4 may have a direct role in the development of the vasculature in the yolk sac, we determined the expression pattern of Lef1 and Alx4 in extraembryonic tissues (Fig. 5). The yolk sac comprises adjacent mesodermal and primitive (visceral) endoderm (VE) cell layers (Palis and Yoder, 2001; Baron, 2003). Lef1 was expressed in cells of the mesoderm as well as endodermal cells of the yolk sac (Fig. 5C). However, we failed to detect Alx4 expression in the yolk sac (Fig. 5A). The amnion, an extra-embryonic extension of the somatopleure, also consists of juxtaposed mesodermal and ectodermal monolayers of flattened cells (Gardner, 1983; Lawson et al., 1991). Here both Lef1 (Fig. 5D) and Alx4 (Fig. 5B) are expressed in the mesoderm, whereas Lef1 is also evident in the ectodermal layer. Taken together, we suggest that the diminished vasculature in the yolk sac arises as an indirect consequence of defective cardiovascular system associated with amnion and/or embryo proper

\section{Defective vasculature in Lef1/\%/Alx $4^{/ s t D / l s t D}$ embryos}

Since mutations affecting blood vessel formation cause embryonic lethality (Rossant and Howard, 2002), we determined the overall structure of the embryonic vasculature by whole-mount IHC detection of the murine vascular endothelial cells marker, PECAM (Fig. 6). Abnormalities of the intersomitic blood vessels or the vessels of the limb buds were not observed in $9.5 \mathrm{dpc}$ compound homozygous embryos (Fig. 6A and C). However, the pattern of the head vasculature was significantly altered. Normally, the sprouting of branches from the cardinal veins form a hierarchical network of large-diameter vessels, the primitive neural plexus, in the medial region in the head (see arrows in Fig. 6B). In contrast, the major
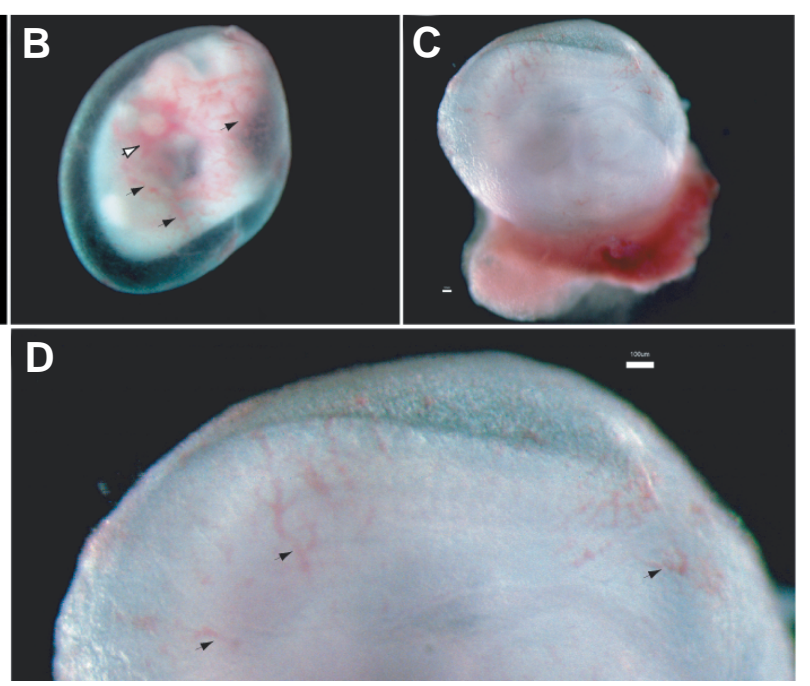


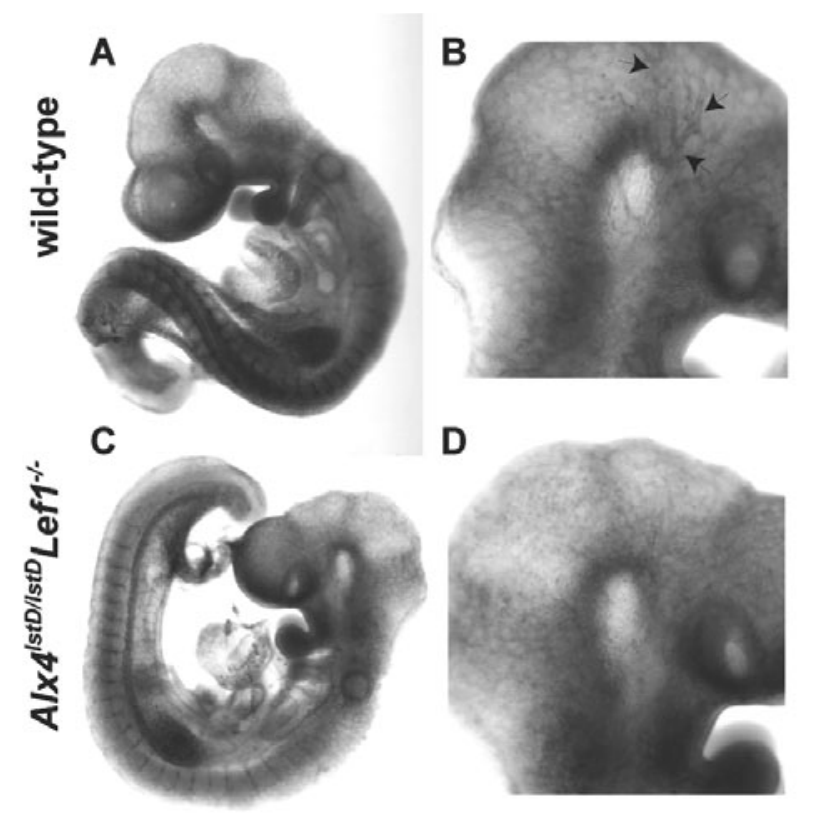

Fig. 6. Vascular development in Lef1 $\% / A I x 4^{I s t D / / s t D}$ embryos. Wholemount immunohistochemistry using anti-PECAM1 antibody, an endothelial marker, was used to visualize vessel development in $9.5 \mathrm{dpc}$ embryos. PECAM staining detects a developed vasculature in both control (A) and in Lef1 $1 /$ Alx $4^{\mathrm{IStD} / \mathrm{IstD}}$ (C) embryos. However, upon closer examination of the head region, the control embryo has a well-developed network of vessels (B). In the compound mutants (D), the vascular network is not as well developed as compared to controls. The vessels are smaller and there is an absence of the major vessels evident in wild type enbryos.

Given the apparent vascular defects in these embryos, detailed analysis of the expression of Alx4 and Lef1 in cells associated with the developing vasculature was performed (Figs. 7,8). Both Alx4 (Fig. 7) and Lef1 (Fig. 8) are strongly expressed in mesenchymal cells surrounding the PE-CAM-positive vascular endothelial cells of the umbilical vein and dorsal aorta, respectively. However, expression of Alx 4 in the nuclei of endothelial cells (arrowheads) is not detected. In the case of Lef1, very weak expression just above background levels is seen in the nuclei of endothelial cells, although this level of expression is significantly reduced relative to Lef1 expression in the cells surrounding the vascular structures. Thus, we conclude that defects in the vasculature arises, at least in part, due to defects originating from the mesenchyme.

Thus, our data establish the overlapping expression of Lef1 and Alx4 in both embryonic and extra-embryonic tissues. Furthermore, in support of our previous observation describing the physical interaction between Lef1 and Alx4 (Boras and Hamel, 2002), we observed a genetic interaction between these two factors as evidenced by the early embryonic lethality and the associated vascular defects in both the yolk sac and the developing embryo. These data reveal, therefore, a previously uncharacterized requirement for the coordinated activities of Lef1 and Alx4 during early embryonic development.

\section{Discussion}

Here we have characterized in mice a genetic interaction between the mediator of Wnt-signaling, Lef1 and the homeodomain factor, Alx4. While deletion of either of these genes individually results in an array of defects in organogenesis, loss of either Lef1 or Alx4 activity is not deleterious to embryo viability (van Genderen etal., 1994; Qu etal., 1997b; Qu etal., 1998; Galceran etal., 1999). All Lef1-deficient mutants are born but die soon after birth, while 2\% of mice deficient for Alx4 activity $\left(A / \times 4^{/ s t D / s t D}\right)$ are viable after birth and are fertile. The majority of $A / \times 4^{\mid s t D / s t D}$ embryos die perinataly from gastrochinesis (ventral body wall closure defect). In contrast, loss of both Lef1 and Alx4 activity unexpectedly resulted in early embryonic lethality at mid-gestation and was associated with abnormalities in vascular patterning of the cranial region. Detailed analysis of embryonic expression of Lef1 and Alx4 revealed that the overlapping functions of Lef1 and Alx4 may be important for vascular development in the head region and may effect indirectly the yolk sac. Accordingly, retarded embryonic growth was evident as early as $9 \mathrm{dpc}$ with lethality occurring in $100 \%$ of embryos by 10 dpc.

Prior to $9.5 \mathrm{dpc}$, viability of murine embryos is sustained by the direct exchange of gases, nutrients and toxic metabolites with the surrounding amnionic fluid (Rossant and Cross, 2001). However, between $8.5 \mathrm{dpc}$ to $11.5 \mathrm{dpc}$, viability is dependent on development of functional yolk sac circulation and its communication with the embryo. Blood vessels in the yolk sac initially form between the outer endodermal and inner mesoderm layers, angioblasts first appearing around 7.0-7.7 dpc. Until the chorioallantoic placenta is established, the yolk sac acts as a primitive placenta, its vasculature having fused with the vasculature of the embryo by $8.5 \mathrm{dpc}$. From approximately $9.5 \mathrm{dpc}$ onwards, the chorioallantoic placenta replaces the yolk sac to support embryonic growth and development. Our data revealed that loss of either Lef1 or Alx4 alone had no discernable effect on vasculogenesis in the yolk sac (data not shown) while impairment in development of a robust vascular network in the yolk sac was observed for $L$ ef $1^{-1 /} / A / \times 4^{\mid s t D /}$ $I s t D$ embryos. While Lef1 is expressed in both endodermal and mesodermal cells of the yolk sac, Alx4 expression is absent in this extra-embryonic tissue. Both Alx4 and Lef1 are expressed in mesenchymal cells of the umbilical vessels connecting the embryo to the yolk sac vasculature. Dilation of the umbilical vessel is apparent in a subset of compound mutants. Thus, we propose that the yolk sac vascular defect may be a secondary effect related to a lack of blood flow between the embryo and yolk sac. This defect potentially arises due to defective vascularization in the connecting amnion/umbilical region and/or in the embryo proper. Similarly, activity of both Lef1 and Alx4 is required for at least some embryonic vasculogenic processes since analysis of the embryonic vascular network revealed defects in the vascular network in the head. In this case, compound homozygous embryos exhibited smaller vessels in the vascular network and an absence of the major vessels in the head.

Loss of Alx4 activity does not appear to effect development of all extra-embryonic tissues for which Lef1 activity is required. The chorioallantoic placenta, for example, develops following interaction between vascularized allantois and the chorionic plate. While this process is dependent, in part, on Lef1 (Galceran et al., 1999), loss simultaneously of Alx4 and Lef1 did not produce any apparent developmental defects, consistent with lack of $A / x 4$ expression in the developing placenta (data not shown).

Lef1 is an important transcriptional mediator of the "canonical" Wnt-signaling pathway. In this pathway, Wnt-signaling causes stabilization of cytoplasmic $\beta$-catenin, resulting in its nuclear 

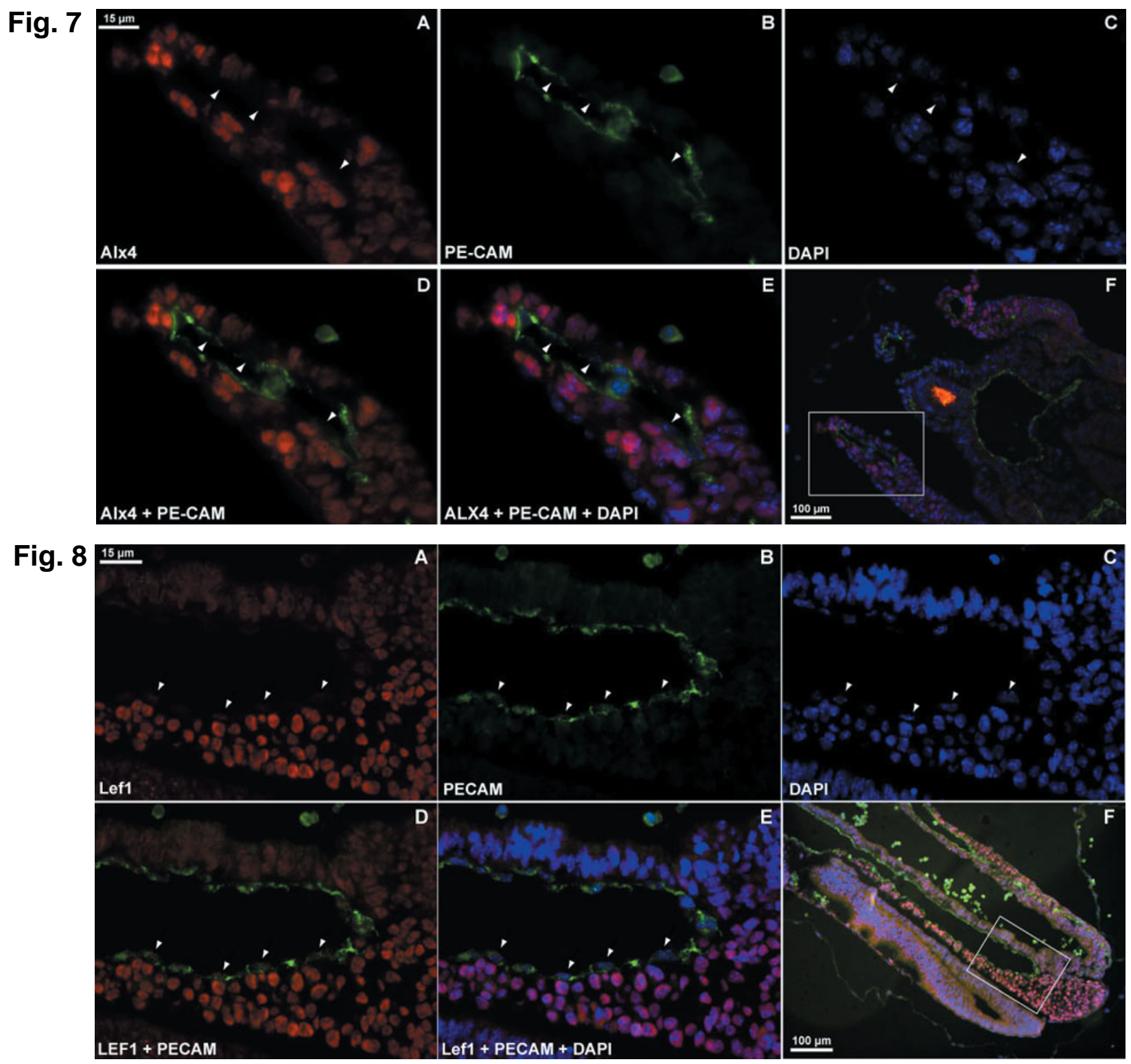

Fig. 7. Transverse sections of 9.5 dpc embryo illustrating co-expression of Alx4 and PE-CAM in mesenchymal cells surrounding the umbilical vein. Alx4 expression (A) is restricted to mesenchymal cells surrounding the umbilical vein while PE-CAM expression (B) marks vascular endothelial cells. Merge of Alx4 and PE-CAM (D) reveals that Alx4 is not expressed in the nuclei of vascular cells, visualized by DAPI staining (C). Alx4, PE-CAM and DAPI are merged in (E). Bars in A-E, $15 \mu \mathrm{m}$. Bars in F, $100 \mu \mathrm{m}$.

Fig. 8. Sagittal section of 9.5 dpc embryo revealing co-expression of Lef1 and PE-CAM in mesenchymal cells adjacent to the dorsal aorta. Lef1 expression (A) is seen in to mesenchymal cells adjacent to the dorsal aorta. It is also weakly expressed in the nuclei of cells expressing PE-CAM (B) (see arrowheads). Merge of Lef1 and PE-CAM (D) reveals that Lef1 is weakly expressed in the nuclei of vascular endothelial cells, visualized by DAPI staining (C). Lef1, PE-CAM and DAPI are merged in (E). Bars in A-E, $15 \mu \mathrm{m}$. Bars in F, $100 \mu \mathrm{m}$.

relocalization to Lef1-containing transcriptional complexes (for review see (Eastman and Grosschedl, 1999)). The role of Wntsignaling in vascular development remains largely uncharacterized. Inactivation of Frizzled 5 (Fzd5), which acts as the receptor for Wnt5a, Wnt10b and Wnt2, causes embryonic lethality due to defective vascular organization in the yolk sac and placenta (Ishikawa et al., 2001). However, these latter Wnts and Fzd5 typically signal using the $\mathrm{Wnt} / \mathrm{Ca}^{++}$pathway, which is distinct from the "canonical" pathway mediated through Lef1 (for example see (van Gijn et al., 2002)). In support of Lef1-dependent role in vasculogenesis are mice deficient for $\beta$-catenin which exhibit altered vascular patterning in the head, vitelline, umbilical vessels and the placenta (Cattelino et al., 2003). Defects in the formation of the placenta were also observed in mice lacking both Lef1 and Tcf1, although no defects in vasculogenesis were apparent (Galceran et al., 1999). These studies support a role where Lef1 activity may play a role in vascular patterning and that Alx4 activity impinges on these Lef1/Wnt-signaling-dependent processes. 

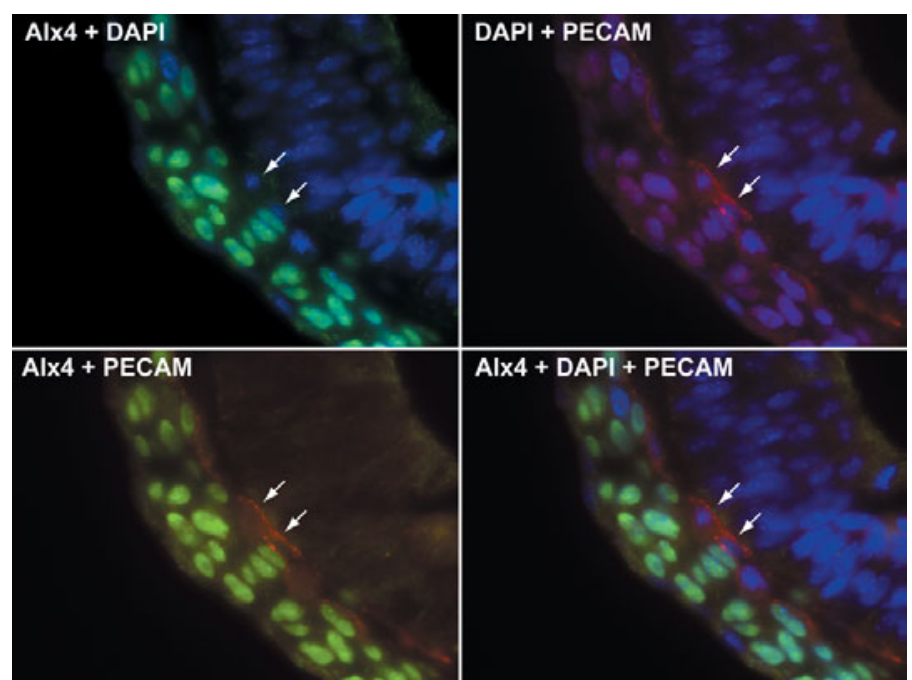

Fig. 9. Lack of overlapping expression of Alx4 and PE-CAM in cephalic region. Transverse section of $9.5 \mathrm{dpc}$ embryo. Simultaneous staining for AlX4 (FITC; green), PE-CAM (rhodamine; red) and nuclei (DAPl; blue). (Lower Left) Co-expression of Alx4 and PE-CAM in mesenchymal cells surrounding the umbilical vein. Alx4 expression is restricted to mesenchymal cells surrounding the umbilical vein while PE-CAM expression marks vascular endothelial cells. Merge of Alx4 and PE-CAM (Lower Right) reveals that Alx4 is not expressed in the nuclei of vascular cells. Arrows point to identical nuclei in all four panels.

The role of Alx4 during vasculogenesis has been suggested previously. A recent report of a family with the human skull disorder, Parietal foramina, which arises due to a mutation the $A \angle X 4$ gene, has suggested a potential role in vascular development (Valente et al., 2004). Given that both Lef1 and Alx4 are expressed in the mesenchyme from which the vasculature is derived, our data supports a role for these factors in development of the vasculature in the developing embryo. It is clear, however, that the role of Alx4 in cephalic vasculature must originate in the surrounding mesenchyme. Specifically, Alx 4 is not expressed in nuclei of vascular endothelial cells, including the cells in the head region (see Fig. 9). Thus, the precise nature of the combinatorial defect arising due to the loss of both Alx4 and Lef1 requires further detailed analysis in order determine the mechanisms by which these factors coordinately regulate normal vascular development.

To conclude, we have demonstrated that the overlapping activities of Alx4 and Lef1 mediate early embryonic development and further support our previous studies demonstrating the physical interaction between Lef1 and Alx4 proteins (Hudson et al., 1998; Boras and Hamel, 2002). Our data predict further that Alx4 may be an important modulator of the canonical Wnt-signaling pathway during specific developmental processes.

\section{Materials and Methods}

\section{Animals and genotyping}

C57BI/6 mice, heterozygous for a knockout allele of Lef1 have been described previously (van Genderen et al., 1994). The DNA-binding defective allele of Alx4, $/ s t^{D}$, from the inbred strain, Strong's luxoid (Forsthoefel et al., 1966; Forsthoefel, 1968) was back crossed (>20 generations) onto $\mathrm{C} 57 \mathrm{BI} / 6$. Mice homozygous for the $/ s t^{D}$ allele pheno- copies mice with a complete knockout of $A / x 4$ (Qu et al., 1997b; Qu et al., 1998). Mice heterozygous for both the null allele of $\operatorname{Lef1}\left(\operatorname{Lef}^{+-}\right)$and the $/ s t D$ allele of $A / \times 4\left(A / \times 4^{+/ s t}\right)$ were then used to generate animals homozygous for both mutant alleles ( $L$ ef $\gamma^{-} / / A / x 4^{\prime s t D / l s t D}$ ). Note that the phenotype of animals homozygous for one mutant allele and heterozygous for the other ( $L e f^{-1 /} / A / x 4^{+/ / s t D}$ and $L e f^{+/-} / A / x 4^{/ s t D / s t D}$ ) gave rise to phenotypes indistinguishable from the individually homozygous ( $L e f 1^{-/}$and $A / \times 4^{/ s t D /}$ $I_{s t D}$, respectively) animals described previously (Forsthoefel et al., 1966; Forsthoefel, 1968; van Genderen et al., 1994; Qu et al., 1998).

Lef1 genotyping has been described previously (van Genderen et al., 1994). For genotyping of the Alx $4 / s t D$ allele, the forward primer in the homeodomain-containing second exon sequence was 5'CAA AGG CAA GAA GCG GCG GAA TC (C>T mutation introduced into primer which allows for Taql digestion) and the reverse primer in second intron sequence was 5'GGT ACA TTG AGT TGT GCT GTC C- $3^{\prime}\left(94^{\circ} \mathrm{C}, 50^{\circ} \mathrm{C}\right.$, $72^{\circ} \mathrm{C}, 30$ cycles). The PCR product was then digested with Taq1 at $65^{\circ} \mathrm{C}$ for $4 \mathrm{hrs}$ and run out on a $4 \%$ agarose gel. The mutant band does not cut and runs at $317 \mathrm{bp}$ while the wild type allele is cut and appears at $295 \mathrm{bp}$ removing $22 \mathrm{bp}$.

\section{Whole-mount in situ hybridization and immunofluorescence}

Embryos were fixed in $4 \%$ paraformaldehyde (PFA) overnight at $4{ }^{\circ} \mathrm{C}$. For whole-mounts, embryos were dehydrated serially to $100 \%$ methanol, treated for 5 hours with 1:5 hydrogen peroxide in methanol and rehydrated to PBST at room temperature. Whole-mount in situhybridizations were performed with digoxigenin-labeled antisense riboprobes as described previously (Motoyama et al., 1998). The cDNA for probes for Lef1 and Alx4 have been described (Hudson et al., 1998). For whole-mount immunohistochemistry, embryos were blocked for 1 hour in blocking reagent (Boerengher Mannheim). After overnight incubation with antiPECAM1 (1:100, BD Biosciences) in blocking solution, embryos were washed 5 times for 1 hour each in PBST at room temperature and treated overnight with secondary antibody (peroxidase-conjugated goat anti-rat, $\mathrm{KPL}$ ) in blocking solution at $4^{\circ} \mathrm{C}$. Peroxidase activity was then visualized using DAB (Vector labs). Immunohistochemical analysis of embryo sections was performed according to the instructions provided in the R\&D HRP-AEC immunostaining kits, with the following amendments. Immunofluorescence was performed on frozen sections. Briefly, $9 \mathrm{dpc}$ embryos were snap-frozen. Frozen sections were rehydrated with PBS for 1 hour and treated with PBS plus $0.1 \%$ Triton $\mathrm{X}-100$ for 5 minutes. Primary antibodies for rabbit Lef1 antisera (Dr. R Grosschedl) and mouse antiAlx4 monoclonal antibody (Hudson et al., 1998) were incubated for 1 hour at room temperature and FITC- or Cy3-labeled secondary antibodies added for 1 hour at room temperature. Slide were mounted with VectaShield mounting media (Vector, Burlingame, CA, USA) impregnated with DAPI. Images of whole mounts were taken by using a Nikon DXM 1200 digital camera driven by Nikon ACT-1 imaging software. Fluorescence images were taken a Nikon Ellipse 80i fluorescence microscope using a $Q$ Imaging Fast 1394 digital camera and compiled using Simple PCl software (Nikon, Canada).

\section{Acknowledgements}

The authors would like to thank members of the PAH lab for their helpful discussions and input on the manuscript. Excellent technical assistance was also provided by Dr. Stuart Yang. This work was funded by a cooperative Canadian Breast Cancer Research Alliance-Canadian Institutes of Health Research grant to PAH from (Grant \# MOP-49614).

\section{References}

ADAMS, R.H., PORRAS, A., ALONSO, G., JONES, M., VINTERSTEN, K., PANELLI, S., VALLADARES, A., PEREZ, L., KLEIN, R. and NEBREDA, A.R. (2000). Essential role of p38alpha MAP kinase in placental but not embryonic cardiovascular development. Mol. Cel/6:109-116. 
AMBLER, C.A., NOWICKI, J.L., BURKE, A.C. and BAUTCH, V.L. (2001). Assembly of trunk and limb blood vessels involves extensive migration and vasculogenesis of somite-derived angioblasts. Dev. Biol. 234:352-364.

BARAK, Y., NELSON, M.C., ONG, E.S., JONES, Y.Z., RUIZ-LOZANO, P., CHIEN, K.R., KODER, A. and EVANS, R.M. (1999). PPAR gamma is required for placental, cardiac and adipose tissue development. Mol. Cel/4:585-595.

BARON, M.H. (2003). Embryonic origins of mammalian hematopoiesis. Exp. Hematol. 31:1160-1169.

BEHRENS, J., VON KRIES, J.P., KUHL, M., BRUHN, L., WEDLICH, D., GROSSCHEDL, R. and BIRCHMEIER, W. (1996). Functional interaction of beta-catenin with the transcription factor LEF-1. Nature 382:638-642.

BORAS, K. and HAMEL, P.A. (2002). Alx4 Binding to LEF-1 Regulates N-CAM Promoter Activity. J. Biol. Chem. 277:1120-1127.

BRUHN, L., MUNNERLYN, A. and GROSSCHEDL, R. (1997). ALY, a contextdependent coactivator of LEF-1 and AML-1, is required for TCRalpha enhancer function. Genes Dev. 11:640-653.

BRUNNER, E., PETER, O., SCHWEIZER, L. and BASLER, K. (1997). pangolin encodes a Lef- 1 homologue that acts downstream of Armadillo to transduce the Wingless signal in Drosophila. Nature 385:829-833.

CARLSSON, P., WATERMAN, M.L. and JONES, K.A. (1993). The hLEF/TCF-1 alpha HMG protein contains a context-dependent transcriptional activation domain that induces the TCR alpha enhancer in T cells. Genes Dev. 7:24182430.

CATTELINO, A., LIEBNER, S., GALLINI, R., ZANETTI, A., BALCONI, G., CORSI, A., BIANCO, P., WOLBURG, H., MOORE, R., OREDA, B., KEMLER, R. and DEJANA, E. (2003). The conditional inactivation of the beta-catenin gene in endothelial cells causes a defective vascular pattern and increased vascular fragility. J. Cell. Biol. 162:1111-1122.

CAVAllo, R.A., COX, R.T., MOLINE, M.M., ROOSE, J., POlEVOY, G.A., CLEVERS, H., PEIFER and M., BEJSOVEC, A. (1998). Drosophila Tcf and Groucho interact to repress Wingless signalling activity. Nature 395:604-608.

DE LAU, W. and CLEVERS, H. (2001). LEF1 turns over a new leaf. Nat. Genet. 28:3-4.

EASTMAN, Q. and GROSSCHEDL, R. (1999). Regulation of LEF-1/TCF transcription factors by Wnt and other signals. Curr. Opin. Cell Biol. 11:233-240.

FORSTHOEFEL, P.F. (1968). Responses to selection for plus and minus modifiers of some effects of Strong's luxoid gene on the mouse skeleton. Teratology $1: 339-351$.

FORSTHOEFEL, P.F., FRITTS, M.L. and HATZENBELER, L.J. (1966). The origin and development of alopecia in mice homozygous for strong's luxoid gene. $J$. Morphol. 118:565-580.

GALCERAN, J., FARINAS, I., DEPEW, M.J., CLEVERS, H. and GROSSCHEDL R. (1999). Wnt3a-/- like phenotype and limb deficiency in Lef1(-/-)Tcf1(-/-) mice. Genes Dev. 13:709-717.

GALCERAN, J., HSU, S.C. and GROSSCHEDL, R. (2001). Rescue of a Wn mutation by an activated form of LEF-1: regulation of maintenance but not initiation of Brachyury expression. Proc. Natl. Acad. Sci. U.S.A. 98:8668-8673.

GALCERAN, J., MIYASHITA-LIN, E.M., DEVANEY, E., RUBENSTEIN, J.L. and GROSSCHEDL R. (2000). Hippocampus development and generation of dentate gyrus granule cells is regulated by LEF1. Development 127:469-482.

GARDNER, R.L. (1983). Origin and differentiation of extraembryonic tissues in the mouse. Int. Rev. Exp. Pathol. 24:63-133.

GIESE, K., AMSTERDAM, A. and GROSSCHEDL, R. (1991). DNA-binding properties of the HMG domain of the lymphoid-specific transcriptional regulator LEF1. Genes Dev. 5:2567-2578.

GIESE, K., COX, J. and GROSSCHEDL, R. (1992). The HMG domain of lymphoid enhancer factor 1 bends DNA and facilitates assembly of functional nucleoprotein structures. Cel/69:185-195.

GIESE, K and GROSSCHEDL, R. (1993). LEF-1 contains an activation domain that stimulates transcription only in a specific context of factor-binding sites. EMBO J12:4667-4676.

GIESE, K., KINGSLEY, C., KIRSHNER, J.R. and GROSSCHEDL, R. (1995). Assembly and function of a TCR alpha enhancer complex is dependent on LEF1 -induced DNA bending and multiple protein-protein interactions. Genes Dev.
9:995-1008

HUBER, O., KORN, R., MCLAUGHLIN, J., OHSUGI, M., HERRMANN, B.G. and KEMLER, R. (1996). Nuclear localization of beta-catenin by interaction with transcription factor LEF-1. Mech. Dev. 59:3-10.

HUDSON, R., TANIGUCHI-SIDLE, A., BORAS, K., WIGGAN, O. and HAMEL, P.A (1998). Alx-4, a transcriptional activator whose expression is restricted to sites of epithelial-mesenchymal interactions. Dev. Dyn. 213:159-169.

ISHIKAWA, T., TAMAI, Y., ZORN, A.M., YOSHIDA, H., SELDIN, M.F., NISHIKAWA, S. and TAKETO, M.M. (2001). Mouse Wnt receptor gene Fzd5 is essential fo yolk sac and placental angiogenesis. Development 128:25-33.

JIN, Z.X., KISHI, H., WEI, X.C., MATSUDA, T., SAITO, S. and MURAGUCHI, A. (2002). Lymphoid enhancer-binding factor- 1 binds and activates the recombination-activating gene-2 promoter together with c-Myb and Pax-5 in immature B cells. J. Immunol. 169:3783-3792.

KRAMPS, T., PETER, O., BRUNNER, E., NELLEN, D., FROESCH, B. CHATTERJEE, S., MURONE, M., ZULLIG, S. and BASLER, K. (2002). Wnt/ wingless signaling requires BCL9/legless-mediated recruitment of pygopus to the nuclear beta-catenin-TCF complex. Ce//109:47-60.

KRATOCHWIL, K., DULL, M., FARINAS, I., GALCERAN, J. and GROSSCHEDL, R. (1996). Lef1 expression is activated by BMP-4 and regulates inductive tissue interactions in tooth and hair development. Genes Dev. 10:1382-1394.

KRATOCHWIL, K., GALCERAN, J., TONTSCH, S., ROTH, W. and GROSSCHEDL, R. (2002). FGF4, a direct target of LEF1 and Wnt signaling, can rescue the arrest of tooth organogenesis in Lef1(-/-) mice. Genes Dev. 16:3173-3185.

LABBE, E., LETAMENDIA, A. and ATTISANO, L. (2000). Association of Smads with lymphoid enhancer binding factor $1 / T$ cell-specific factor mediates cooperative signaling by the transforming growth factor-beta and wnt pathways. Proc. Nat/. Acad. Sci. USA 97:8358-8363.

LAWSON, K.A., MENESES, J.J. and PEDERSEN RA. (1991). Clonal analysis of epiblast fate during germ layer formation in the mouse embryo. Development 113:891-911.

LEVANON, D., GOLDSTEIN, R.E., BERNSTEIN, Y., TANG, H., GOLDENBERG, D., STIFANI, S., PAROUSH, Z. and GRONER, Y. (1998). Transcriptional repression by AML1 and LEF-1 is mediated by the TLE/Groucho corepressors. Proc. Natl. Acad. Sci. USA 95:11590-11595.

MOTOYAMA, J., HENG, H., CRACKOWER, M.A., TAKABATAKE, T., TAKESHIMA K., TSUI, L.C. and HUI, C. (1998). Overlapping and non-overlapping Ptch2 expression with Shh during mouse embryogenesis. Mech. Dev. 78:81-84.

PALIS, J. and YODER, M.C. (2001). Yolk-sac hematopoiesis: the first blood cells of mouse and man. Exp. Hematol. 29:927-936.

QU, S., LI, L. and WISDOM, R. (1997a). Alx-4: cDNA cloning and characterization of a novel paired-type homeodomain protein. Gene 203:217-223.

QU, S., NISWENDER, K.D., JI, Q., VAN DER MEER, R., KEENEY, D., MAGNUSON, M.A. and WISDOM, R. (1997b). Polydactyly and ectopic ZPA formation in Alx4 mutant mice. Development 124:3999-4008.

QU, S., TUCKER, S.C., EHRLICH, J.S., LEVORSE, J.M., FLAHERTY, L.A., WISDOM, R. and VOGT, T.F. (1998). Mutations in mouse Aristaless-like4 cause Strong's luxoid polydactyly. Development 125:2711-2721.

QU, S., TUCKER, S.C., ZHAO, Q., DECROMBRUGGHE, B. and WISDOM, R (1999). Physical and genetic interactions between Alx4 and Cart1. Development 126:359-369.

ROOSE, J., MOLENAAR, M., PETERSON, J., HURENKAMP, J., BRANTJES, H., MOERER, P., VAN DE WETERING, M., DESTREE, O. and CLEVERS, H. (1998). The Xenopus Wnt effector XTcf-3 interacts with Groucho-related tran scriptional repressors. Nature 395:608-612.

ROSSANT, J. and CROSS, J.C. (2001). Placental development: lessons from mouse mutants. Nat. Rev. Genet. 2:538-548.

ROSSANT, J. and HOWARD, L. (2002). Signaling pathways in vascular development. Annu Rev Cell Dev. Biol.18:541-573.

TRAVIS, A., AMSTERDAM, A., BELANGER, C. and GROSSCHEDL, R. (1991). LEF-1, a gene encoding a lymphoid-specific protein with an HMG domain, regulates T-cell receptor alpha enhancer function. Genes Dev. 5:880-894.

VALENTE, M., VALENTE, K.D., SUGAYAMA, S.S. and KIM, C.A. (2004). Malformation of cortical and vascular development in one family with parietal foramina 
610 K. Boras-Granic et al.

determined by an ALX4 homeobox gene mutation. AJNR Am. J. Neuroradiol. 25:1836-1839.

VAN GENDEREN, C., OKAMURA, R.M., FARINAS, I., QUO, R.G., PARSLOW, T.G., BRUHN, L. and GROSSCHEDL, R. (1994). Development of several organs that require inductive epithelial-mesenchymal interactions is impaired in LEF-1-deficient mice. Genes Dev. 8:2691-2703.

VAN GIJN, M.E., DAEMEN, M.J., SMITS, J.F. and BLANKESTEIJN, W.M. (2002). The wnt-frizzled cascade in cardiovascular disease. Cardiovasc. Res. 55:1624.

WILSON, D., SHENG, G., LECUIT, T., DOSTATNI, N. and DESPLAN, C. (1993). Cooperative dimerization of Paired class homeodomains on DNA. Genes Dev. 7:2120-2134.
WILSON, D.S. and DESPLAN, C. (1995). Homeodomain proteins. Cooperating to be different. Curr. Biol. 5:32-34.

WILTING, J., BRAND-SABERI, B., HUANG, R., ZHI, Q., KONTGES, G., ORDAHL, CP., CHRIST, B. (1995). Angiogenic potential of the avian somite. Dev. Dyn. 202:165-171.

Received: February 2006

Reviewed by Referees: April 2006

Modified by Authors and Accepted for Publication: May/June 2006

Published Online: August 2006 